\title{
Role of Gender in Children's Cognitive Learning from Television
}

\section{Yasmeen Sultana ${ }^{1 *}$ and Khushboo Rafiq ${ }^{2}$}

${ }^{1}$ Department of Media and Communication Studies, Sindh Madressatul Islam University, Karachi, Pakistan

${ }^{2}$ Department of Mass Communication, University of Karachi, Karachi, Pakistan

\begin{abstract}
This research examines the gender differences among children in cognitive learning from television with a special focus on vocabulary and perception building. 60 female and 60 male children aged between 7 to 12 years from different schools of Karachi were selected through random sampling. 10 sequential experiments were conducted in those schools' laboratories. Selected animated cartoon episodes were shown to children in each session. After which, children's responses were evaluated by means of questionnaires measuring vocabulary and perception tests. Results showed that female participants performed slightly better than male participants in both tasks. However, this association might have occurred due to chance.
\end{abstract}

Keywords: Gender differences; Vocabulary; Perception; Media effects; Children; Television

\section{Role of Gender}

Role of gender is main phenomenon's that have been studied far and wide by researchers interested in exploring media effects on children [1-5].

A number of studies have revealed that media exposure, particularly learning from television has an undeviating effect on children's behavioural and cognitive abilities. Gerbner in his cultivation theory argues that the more the exposure to media content, the more effected are the people in long term. Gradually, they might started to think and behave accordingly. However, several other factors might also have involved in shaping cognitive skills and attitudes from media exposure. In this regard, gender differences have been an interesting topic throughout different eras in history to ponder on. Eckert and McConnell-Ginet enlighten that gender characteristics are studied not only to classify people and provide them identities, but to maintain social relations.

Majority of scholars believe that gender is a variable which keeps on changing its description in different times. Geographical conditions also play a pivotal role in shaping gender based attitudes. The notion 'gender role' has been evolved and it has different meanings in diverse cultures. Social institutions such as family, neighbourhood and schools etc. are involved in the process of assigning and implementing gender-specific roles. The environment to which a child exposed to in early years, inculcates the gender roles in her/his mind. Kane argues that parents start to categorize children's roles based on the specific characteristics they have in their minds for any particular gender. Consequently, children start to develop their own concepts of gender differences aligned with the norms set by their parents and community.

Apart from the close relationships, the source which might have proved to be persuasive in developing specific gender roles in children's mind is media in general and television in particular. Numerous studies highlight that children store bits of images in their minds whatever they watch on television, and thus act accordingly in their real lives [6,7].

Boys start to believe that they are superior to girls and girls are convinced that they might have created to accomplish certain duties. Ultimately their daily activities begin to revolve around these genderbased stereotypes, which gradually lead them to perform in a predefined way to achieve certain tasks.
However, the results can also be vice-versa if the opposite side of coin would be shown to the television audiences. As Renzetti and Curran mentioned that gender stereotypes can be diminished by displaying both sexes in non-traditional roles. Fairy tales like Cinderella and Snow White and Seven Dwarfs convince viewers to believe that only beautiful and slim girls can find their destiny. But on the other hand, hosts like Oprah Winfrey are also shown on television who has won the hearts of millions of people despite being black and averagelooking. Here an important question rises. Do these images that media create in people's minds also have an effect on their cognitive skills?

\section{Current scenario in Pakistan}

The fraction of school going boys is more than the ratio of school going girls in Pakistan. According to The World Almanac and Book of Facts 2016, 33\% of Pakistani population is consisted of children under 15 years of age. Although, trends are gradually changing but still there are many families even in the urban areas of Pakistan where discriminatory attitude towards girls is in common practice. However, girls at primary level in Pakistan have proved themselves somehow better than boys in many academic tasks just like other countries in the world [8-10]. But there are still many walks of life, where active participation of girls are not appreciated due to gender biasness. This practice can be majorly seen in middle and lower middle socioeconomic backgrounds even in urban areas of Pakistan.

\section{Present study}

The primary aim of this research was to find out if there are any gender differences in vocabulary learning and perception building of children from television. Moreover, to estimate if there is a role of anti-discriminatory gender content in designing the thinking and perception building of female children.

*Corresponding author: Yasmeen Sultana, Department of Media and Communication Studies, Sindh Madressatul Islam University, Karachi, Pakistan, Tel: +921-333-2175865; Fax: +92 21 99217504; E-mail: yasmeensultana@gmail.com, yasmeensultana@smiu.edu.pk

Received March 22, 2018; Accepted April 19, 2018; Published April 23, 2018

Citation: Sultana Y, Rafiq K (2018) Role of Gender in Children's Cognitive Learning from Television. J Mass Communicat Journalism 8: 371. doi: 10.4172/21657912.1000371

Copyright: @ 2018 Sultana $Y$, et al. This is an open-access article distributed under the terms of the Creative Commons Attribution License, which permits unrestricted use, distribution, and reproduction in any medium, provided the original author and source are credited. 
This study is beneficial for analysing children's responses to the screen and determining their cognitive skills from gender's perspective. Ultimately, it will be helpful for child psychologists, parents, school administrations, curriculum designers and media practitioners.

\section{Research questions}

Two questions were tried to ponder upon through this study:

RQ1: Which gender performs better in cognitive learning, such as vocabulary and perception building tasks from television?

RQ2: Is anti-discriminatory gender content helpful in instilling confidence among girls about their own image?

\section{Hypotheses}

Since a female cartoon character was portrayed as a protagonist in the selected content, the hypothesis designed on the basis of above questions is:

H1: Female children perform better on vocabulary tasks than male children.

$\mathrm{H} 2$ : Female children have a better perception and understanding of message than male children.

\section{Method \\ Participants}

The sample size consisted of 60 female and 60 male children, randomly selected from four different schools in diverse populated areas of Karachi, Pakistan. All participants belonged to the age group of 7 to 12 years, which is denoted as concrete operational stage.

\section{Design}

Gender was the independent variable in the study which had two groups, female children and male children whereas vocabulary and perception were the dependent variables. Control variables were message (content), location (Karachi, Pakistan), time period (10 weeks) and laboratories' environment.

\section{Setting and apparatus}

One room designated as computer laboratory in each school was used for this study to play cartoon movies.

These resources were utilized to conduct the study:

\section{Physical Resources:}

LCD Screen (24 inches, SONY TV), 10 Cartoon Movies, Laboratories and Questionnaires.

\section{Personal Resources:}

School contacts and one assistant to help out in distributing and collecting questionnaires.

\section{Selection of Content}

The content chosen for this experiment was ten random episodes of the cartoon series "Meena ke saath" in Urdu language. Meena is a fictional cartoon of a nine years old girl developed by UNICEF during 1990s. The content is almost two decades old. That is why none of the children had seen it before. Cartoon movies chosen for the experiments were "Count your chickens", "Dividing the mango", "Say no to dowry", "Take care of girls", "Saving a life", "Who is afraid of the bully?", "Who is afraid of the bully?", "Will Meena leave school?", "Meena's three wishes", "Too young to marry" and "I love school". The approximate length of each episode was from 11 to 12 minutes, anyhow one cartoon movie, "Meena's three wishes" was just 8 minutes long.

\section{Procedure}

A pilot experiment was conducted before the actual study. In the light of the outcome, some minor changes were done in the multiplechoice options like a few words were replaced with their simpler alternatives, so that children could understand the questions more easily. Moreover, computers were replaced with an LCD screen, Sony 24 inches as computer screen was not clearly visible to all children. Researcher carried this screen in each experiment. After applying these measures, actual study was initiated on alternative weekdays in different schools during the first three months of 2017.

Laboratory experiments were conducted to observe children. First of all, groups comprised of 20 students in each class watched a cartoon episode in every session. There were total 6 groups from 4 different schools, hence overall 120 students participated in the study. 10 individual sessions were performed in each school and questionnaires related to cartoon stories were filled out by the children in order to understand their perception and comprehension of the given message. Later on, filled questionnaires were divided on the basis of gender and differences were evaluated in the light of responses.

\section{Questionnaire design}

Responses were being tested on the basis of a total of 60 multiplechoice questions, out of which 30 questions belonged to vocabulary measurement, whereas other questions were included to estimate children's perception. There were total 10 questionnaires included in the study; each of them was comprised of a separate cartoon movie. Questionnaires were prepared in Urdu language. All questions were related to the cartoon movies shown to the children. Cronbach's Alpha (K-R 20) test was applied to check the reliability of questionnaires. Output indicated that internal consistency was good for both measures (.82 for vocabulary and .83 for perception tests).

\section{Limitations and delimitations}

- Electricity load shedding had been a big issue during experiment. It was tried to select those particular time slots for showing cartoon movies to children when electricity was available.

- Content of two stories "Say no to dowry" and "Too young to marry" were considered inappropriate by one school's administration, although it had been prepared after an extensive and comprehensive research. As a result, the school had been skipped and an alternate school was selected to conduct the study.

\section{Results}

The IBM Statistical Package for Social Sciences (SPSS), version 22 was utilized to evaluate data. Between-subjects design experimental method was chosen to compare both groups. The sample was divided according to gender ( $\mathrm{n}=200,100$ female and 100 male respondents).

Kolmogorov Smirnov Goodness-of-Fit Test was applied to check the nature of our data. The test showed that our data were nonparametric. So certain tests were implemented to evaluate data.

\section{For vocabulary Results}

A chi-square test for association was performed between gender 
and vocabulary. All expected cell frequencies were greater than five. The minimum expected count was 14 . Results indicated that there was not a statistically significant association between gender and vocabulary score, $\chi^{2}(2)=0.167, p=.920$. A Cramer's V test comprised of the numbers of right and wrong answers of all 200 children was conducted to check the difficulty level of both tasks. It recommended that there was a moderately weak association between gender and vocabulary score, Cramer's V=0.029, $p=.920$. In addition, a MannWhitney $U$ test was executed to determine if there were differences in vocabulary score between both genders. Visual assessment of the distributions suggested that vocabulary scores for both groups were dissimilar. However, vocabulary scores for female children (mean rank $=104.44$ ) were statistically higher than for male children (mean rank $=96.56), U=4605.500, z=-0.966, p=0.334$. On vocabulary tests, $13 \%$ were low scorers, $36 \%$ were medium scorers and $51 \%$ were high scorers among girls. Whereas, $15 \%$ boys were low scorers, $35 \%$ were medium scorers and $50 \%$ were high scorers. Figure 1 to observe the categories of low, medium and high scorers of vocabulary tasks in both groups graphically.

\section{For Perception Results}

In the second task, gender was the independent variable and perception was the dependent variable. A chi-square test for association was implemented between both variables. All expected cell frequencies were greater than five. The minimum expected count was 13. Results revealed that there was not a statistically significant association between gender and perception score, $\chi^{2}(2)=4.447, p=.108$. A Cramer's $\mathrm{V}$ test comprised of the numbers of right and wrong answers of all 200 children was performed to determine the difficulty level of both tasks. It suggested that there was a moderately weak association between gender and vocabulary score, Cramer's V $=0.149, p=.108$. Furthermore, a Mann-Whitney $U$ test was conducted to observe if there were any differences in perception scores of both groups. Visual assessment of the distributions showed that perception scores of female and male children were not similar. Nonetheless, perception scores for female respondents (mean rank=105.46) were statistically higher than for male respondents (mean rank=95.54), $U=4504.500, z=-1.214, p=0.225$.

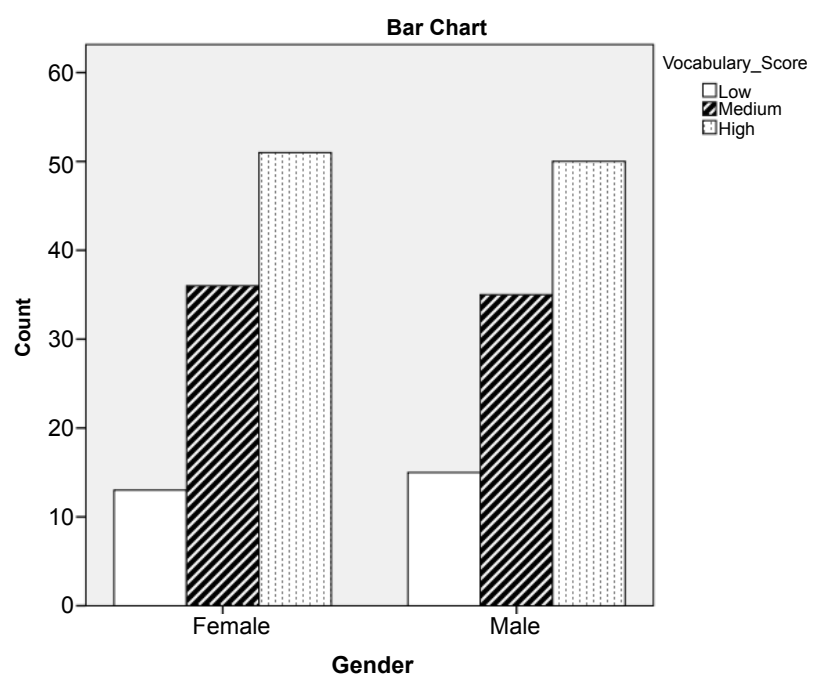

Figure 1: Clustered bar chart comparing vocabulary scores of female and male participants. Here low score is $(<$ or $=50 \%)$, medium score is $(>50 \%$ \& $<80 \%$ ), and high score is (=or $>80 \%$ ).
On perception tests, $8 \%$ were low scorers, $37 \%$ were medium scorers and $55 \%$ were high scorers among girls. On the other hand, $18 \%$ boys were low scorers, $32 \%$ were medium scorers and $50 \%$ were high scorers. Figure 2 to perceive the ranking description and graphical representation in both groups.

By concluding all results, it can be determined that female children performed slightly better than male children. Table 1 for the comparison of mean ranks. Overall, results proved both hypotheses which state that female children perform better on vocabulary and perception tasks than male children. But the differences might have occurred due to chance as indicated by Chi-square test for association.

\section{Discussion}

This study was conducted to determine the gender differences between female and male children in the assessment of vocabulary and perception learning from television. Results demonstrated that girls were slightly better than boys in both tasks.

If we talk about vocabulary tasks, Hyde and Linn [11] presented a meta-analysis of 165 studies which indicated that female accomplished verbal tasks better to some extent than males but the difference was negligible most of the times. Another study by Lowe et al. indicated a little female superiority in memory and verbal abilities when they tested a large sample of more than 1200 children and adolescents on some subsets of TOMAL (Test of Memory and Learning). Scholars such as Burman et al. [12]; Gurian [13] explored the sex differences in neural processing of language among children. They highlighted that girls and

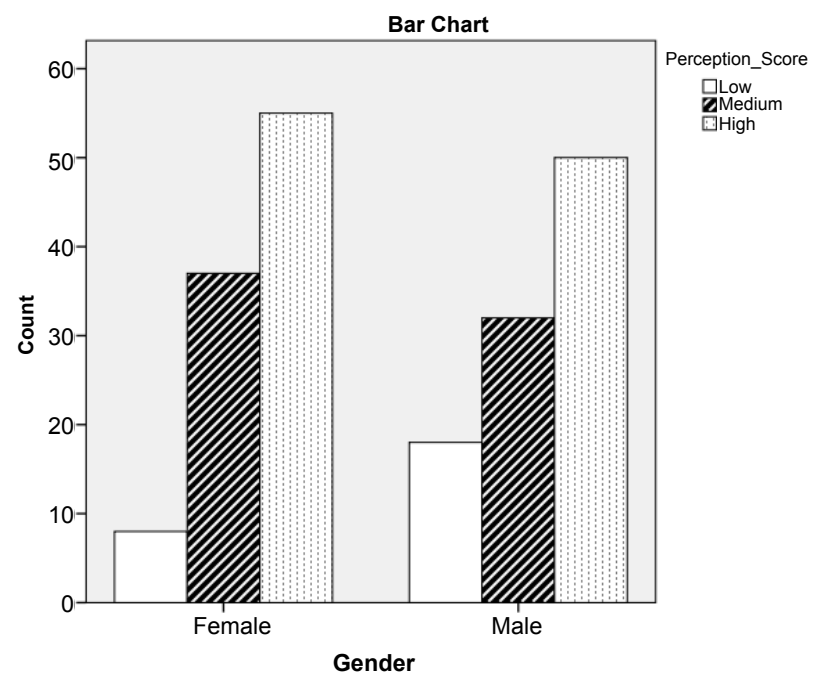

Figure 2: Clustered bar chart comparing perception scores of female and male participants. Here low score is (< or $=50 \%)$, medium score is $(>50 \%$ and $<80 \%$ ), and high score is ( $=$ or $>80 \%$ )

\begin{tabular}{|c|c|c|c|c|}
\hline & \multicolumn{4}{|c|}{ Cognitive Skills } \\
\hline & \multicolumn{3}{|c|}{ Vocabulary } & \multicolumn{2}{c|}{ Perception } \\
\hline Gender & M & SD & M & SD \\
\hline Female & 76.8 & 16.79 & 78.13 & 15.26 \\
\hline Male & 73.70 & 19.27 & 74.07 & 18.87 \\
\hline M & 75.28 & 180.10 & 76.10 & 17.24 \\
\hline
\end{tabular}

Note: Scores represent mean proportions of correct answers and their standard deviations.

Table 1: Group Mean Scores for Vocabulary and Perception as a Function of Gender and Cognitive Skills. 
boys process language tools differently in their minds. According to the research, girls depend on a supramodal language network whereas visual and auditory senses work differently in case of boys. Women often excel at language-based tasks for two reasons: two brain areas that deal with language are larger in females, and females process language in both hemispheres while males favour a single brain half.

Many studies demonstrate that along with biological reasons parents, teachers, and mass media especially television also have a huge impact on children's minds. If we talk about television programs, there are certain characters which are broadcasted to portray heroic tasks, and consequently sometimes create a lasting impact on children's perception of the world.

One reason of slightly better performance of girls over boys might be the content shown to the children. Linebarger noted that young children may imitate what they learn from other persons or characters. The leading character portrayed to children in the study was a nine year old girl Meena, who might had inspired girls more than boys during the sessions. Probably, girls were able to relate many characteristics told verbally or shown in Meena's stories to the incidents similar to their own lives. As in the second episode, Meena's mother gave more food to Raju (Meena's brother) than her. It is a common practice in many households in our population that males are offered the best part of food whereas females have leftovers.

Paul Gilbert in his book "The Compassionate Mind" wrote that self-consciousness and imitation are directly linked to cognition. People usually remember the characteristics of those whom they want to be in their real lives. Such remembrance may have a powerful effect in shaping their behaviours and determining their cognition.

Klein and Shiffman pointed out those animated cartoons are usually gender biased and created specific gender roles in children's minds. Witt [14] supported the idea by reviewing several studies on the influence of television on children's gender role socialization and reached on the conclusion that television is a powerful tool in shaping children's traditional view towards gender biased roles. Nolan et al. indicated that "Children are covertly taught on television that boys are more significant persons than girls."

On contrary, a female child had a leading role in our study, so it can be estimated that females might took more interest and developed some more understanding of the content than male participants because of the empathy they experienced during the sessions.

Luecke-Aleksa et al. compared the association between gender constancy and television viewing. After analysing 313 diaries of television viewing habits of 5 years old children, they anticipated that gender constant children were biased towards characters portrayed on television as compared to those who did not have any concept of gender identity. These results demonstrate that portrayal of a particular gender as an active agent of society might shape a positive attitude towards that gender. Renzetti and Curran also supported the idea.

If we discuss about the psychological aspect of the results, Jean Piaget might be the most relevant theorist to quote here. He did not mention any separate gender differences after conducting several experiments on children, but assumed that age might be the main factor to estimate cognitive differences among children.

Nature versus nurture may also have some effect while discussing the slightly better performance of girls over boys in vocabulary and perception. Hyde [15] emphasized in the light of a meta-analysis on more than hundred studies on gender differences over three million people that there were not any distinctive gender differences in estimating cognitive abilities. Certain age groups and test methods were found to be particularly suitable for analysing specific cognitive skills. Feingold mentioned that girls performed somehow better in the tests of grammar, spelling, and perceptual speed, whereas boys scored a bit higher when tested for spatial visualization, high school mathematics, and mechanical aptitude. No distinct gender differences were observed in overall cognitive skills.

All in all, it can be assumed that probably the selection of gender specific content, brain mechanism and particular test measurements are some reasons of a slight better performance of girls over boys.

\section{Conclusion}

The study proposes the idea to be true that pro-feminine or gender neutral content might be helpful to enhance the confidence of female children. Such content might also be supportive to draw a positive image of female role in the minds of male children. Girls were found to be a little better than boys in verbal and perceptual tasks, but the difference was almost negligible.

\section{Acknowledgements}

This study was not supported by any grant. The authors are grateful to all students who participated in the study, and to the school authorities who allowed them to conduct the experiment in their schools' premises.

\section{Recommendations}

This study can be replicated by taking other independent variables related to cognitive skills, such as memory, pattern recognition and speed of information processing. It will be helpful to find out if there are similar differences exist between both sexes in other skills of cognition. Consequently, particular television content can be prepared for children from both sexes according to their specific areas of interests.

\section{References}

1. Buckingham D (1993) Children Talking Television: The Making of Television Literacy: Psychology Press. UK, p: 321.

2. Cherney ID, London K (2006) Gender-linked differences in the toys, television shows, computer games, and outdoor activities of 5-to 13-year-old children. Sex Roles 54: 717

3. Collins RL (2011) Content analysis of gender roles in media: Where are we now and where should we go? Sex Roles 64: 290-298.

4. Hargittai E, Shafer S (2006) Differences in actual and perceived online skills The role of gender. Social Science Quarterly 87: 432-448.

5. Riding R, Grimley M (1999) Cognitive style, gender and learning from multimedia materials in 11year old children. Br J Educ Technol 30: 43-56.

6. Crawley AM, Anderson DR, Santomero A, Wilder A, Williams M, et al. (2002) Do Children Learn How to Watch Television, The Impact of Extensive Experience With Blue's Clues on Preschool Children's Television Viewing Behavior. J Commun 52: 264-280.

7. Field DE, Anderson DR (1985) Instruction and modality effects on children's television attention and comprehension. J Educ Psychol 77: 91-100.

8. Correa-Burrows P, Burrows R, Ibaceta C, Orellana Y, Ivanovic D (2017) Physically active Chilean school kids perform better in language and mathematics. Health Promot Int 32: 241-249.

9. Fischer F, Schult J, Hell B (2013) Sex differences in secondary school success: why female students perform better. Eur J Psychol Assess 28: 529-543.

10. Stevenson DL, Baker DP (1987) The family-school relation and the child's school performance. Child development 58: 1348-1357.

11. Hyde JS, Linn MC (1988) Gender differences in verbal ability: A meta-analysis. American Psychological Association 104: 53-69. 
Citation: Sultana Y, Rafiq K (2018) Role of Gender in Children's Cognitive Learning from Television. J Mass Communicat Journalism 8: 371 . doi: 10.4172/2165-7912.1000371

Page 5 of 5

12. Burman DD, Bitan T, Booth JR (2008) Sex differences in neural processing of language among children. Neuropsychologia 46: 1349-1362.

13. Gurian M (2010) Boys and girls learn differently! A guide for teachers and parents: Revised 10th anniversary edition. John Wiley \& Sons.
14. Witt SD (2000) Review of Research: The Influence of Television on Children's Gender Role Socialization. Childhood Education 76: 322-324.

15. Hyde JS (2005) The gender similarities hypothesis. Am Psychol 60: 581-592. 